\title{
REVUE GENERALE SUR LES RHINOLITHIASES : A PROPOS DE 20 CAS
}

\author{
S. KHAROUBI \\ FACULTE DE MEDECINE, ANNABA, ALGERIE.
}

\begin{abstract}
RESUME
Cette étude rapporte 20 cas de rhinolithiases colligés entre 1990 et 2007.

La population concernée surtout des adultes (55\% de sexe féminin) ayant le plus souvent des conditions socio-économiques défavorables.

Le tableau clinique est simple et non spécifique : rhinite obstructive purulente unilatérale et fétide. Des présentations déroutantes sont possibles mais rares (infection sinusienne, oculo-palpébrale).

Le diagnostic repose sur l'examen endonasal notamment à l'optique rigide.

Le scanner est très intéressant pour un bon bilan loco-régional permettant en particulier de reconnaître les lésions associées ou sous-jacentes.

L'extraction par les voies naturelles le plus souvent sous anesthésie locale était le procédé thérapeutique habituel.

Les rhinolithes avaient entre 5 et $50 \mathrm{~mm}$ de diamètre avec seulement 3 corps étranger associés (15\%).

Les complications sont rares (épistaxis : $10 \%$ ) et l'évolution était le plus souvent favorable.

MOTS CLES : Corps étranger nasal, rhinolithiase, rhinorrhée unilatérale fétide.
\end{abstract}

This study reports 20 cases of rhinolithiasis treated between 1990 and 2007 . The population concerns especially adults. The symptoms are generally represented by unilateral purulent rhinorrhea and nasal obstruction. The diagnosis is based on clinical examination. The CT-scan precises extension and associated or underlying lesions. The extraction by natural ways, often under local anesthesia, was proceeded as habitual therapeutic. The rhinoliths had between 5 and $50 \mathrm{~mm}$ of diameter with only 3 foreign bodies associated (15\%). The complications are rare (nasal bleeding: 10\%).

KEY WORDS : Rhinolithiasis, nasa foreign body, purulent rhinorrhea.

\section{INTRODUCTION}

La rhinolithiase se définit comme étant une concrétion calcique solide par dépôt progressif de sels calcaires autour d'une fondation centrale résorbable ou non de forme et de dimensions variables (1).

C'est une pathologie rare notamment dans les pays occidentaux où elle est souvent décrite à titre de curiosité pathologique.

Elle demeure d'actualité dans les pays du tiers monde : Afrique et pays du pourtour Méditerranéen (1, 2, 3, 4, 5) compte tenu de plusieurs facteurs : pollution de l'environnement, fréquence des corps étrangers de la sphère $O R L$ surtout chez l'enfant, certaines habitudes ethno-culturelles (applications endonasales de substances phytothérapiques où dans le cadre de certains rites), couverture sani- taire faible voire un morphotype nasal particulier (valve nasale, volumétrie endonasale) susceptible de perturber la physiologie muco-ciliaire des fosses nasales (rôle aggravant des infections à répétition).

Le rhinolithe est un mélange d'eau (2,9 à 5,9\%), de phosphate de magnésium $(19,46 \%)$, carbonate de calcium $(20,69 \%)$, phosphate de calcium $(44,7 \%)$ et de composés organiques (13,2\%) (6).

La rhinolithiase réalise le tableau banal d'une rhinite obstructive à répétition non spécifique parfois fétide ou sanguinolente. Elle peut néanmoins simuler une pathologie plus grave (une tumeur naso sinusienne) ou être à l'origine d'une complication morbide (infection oculo-palpébrale). Le diagnostic repose sur l'examen endonasal à l'optique rigide complété par une tomodensitométrie des cavités 
nasales.

Le traitement est simple (extraction) et les suites le plus souvent favorables.

\section{MATÉRIEL ET MÉTHODES}

Nous avons colligé 20 observations de rhinolithiases durant la période allant de janvier 1990 a avril 2007.

Le motif de consultation est variable, le plus souvent une rhinorrhée unilatérale chronique et fétide.

Après anamnèse tous les patients ont bénéficié d'un examen clinique complet notamment une endoscopie nasale à l'optique rigide après anesthésie locale à la xylocaine naphtazolinée à $5 \%$ et aspirations nasales.

Un bilan radiologique est systématique: radiographies standards (incidence de Blondeau et crâne de profil) et pour certains patients un scanner des cavités naso sinusiennes. Une fois le bilan loco-régional clinique et radiologique fait l'extraction du rhinolithe est réalisée le plus souvent sous anesthésie locale.

Une désinfection locale est prescrite pendant 8 jours.

Un contrôle clinique est fait après 30 jours, 3 mois et 6 mois.

\section{RESULTATS}

Vingt patients se répartissaient en 6 hommes et 14 femmes soit un sex-ratio de 2,33.

L'âge moyen était de 31,2 ans avec des extrêmes entre 7 et 61 ans.

La majorité de nos patients étaient d'origine rurale (12/20:60\%) avec des conditions socio-économiques médiocres.

Les délais de consultations sont difficiles à préciser.Ce délai fut inconnu dans 12 cas. II variait entre 6 mois et 8 ans (moyenne : 3,6 ans) pour les 8 cas restants (Tableau I).

La sémiologie d'appel est assez univoque : rhinorrhée chronique rebelle à tout traitement et fétide. II s'y associe souvent une obstruction nasale, plus rarement une épistaxis ou des douleurs crânio-faciales. (Tableau I)

Dans un cas il s'agissait d'un examen de principe au cours du bilan d'une adénopathie sus claviculaire fistulisée.

L'examen rhinologique est l'étape fondamentale du diagnostic : au spéculum nasal ou à l'optique rigide. II visualise le rhinolithe (formation grisâtre irrégulière à surface spiculée de consistance pierreuse) son siège et permet un bilan précis des cavités nasales. Le siège des rhinolithes était à droite dans 12 cas (60\%) et à gauche dans 7 cas $(35 \%)$. Dans un cas le rhinolithe était bilatéral par perforation septale $(5 \%)$. (Tableau I)

\begin{tabular}{|c|c|c|c|c|c|c|c|}
\hline $\begin{array}{l}\text { CAS } \\
\text { ANNEE }\end{array}$ & $\begin{array}{l}\text { AGE } \\
\text { (Ama) } \\
56 \times 5 \\
\text { (FiM) }\end{array}$ & $\begin{array}{l}\text { DELNA } \\
\text { DE } \\
\text { SEJOUR }\end{array}$ & $\begin{array}{l}\text { SIORIES } \\
\text { CUNiQuES }\end{array}$ & SIEOE & $\begin{array}{l}\text { RHiNCLITHE } \\
\text { Menturat orz } \\
\text { Coppe etranger }\end{array}$ & $\begin{array}{l}\text { SNES: } \\
\text { TAESTE }\end{array}$ & SUITES \\
\hline 1990 & $\begin{array}{c}40 \\
F\end{array}$ & ? & ON & G & $\begin{array}{l}7 \mathrm{~mm} \\
\text { perle }\end{array}$ & AL & , \\
\hline $\begin{array}{c}2 \\
1991\end{array}$ & $\frac{32}{M}$ & ? & Asymptomatique & G & $12 \mathrm{~mm}$ & AL & 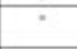 \\
\hline $\begin{array}{c}3 \\
1992\end{array}$ & $\begin{array}{c}17 \\
F\end{array}$ & $?$ & FFF & G & $\begin{array}{l}2 \text { thinolthes } \\
\text { de } 5 \mathrm{~mm}\end{array}$ & AL & - \\
\hline $\begin{array}{c}4 \\
1995\end{array}$ & $\begin{array}{l}13 \\
M\end{array}$ & $\begin{array}{c}\text { Jeune } \\
\text { Age }\end{array}$ & RF & D & $\begin{array}{c}25 \mathrm{~mm} \\
\text { metalique (via) }\end{array}$ & AL. & - \\
\hline $\begin{array}{c}5 \\
1908\end{array}$ & $\begin{array}{l}14 \\
F\end{array}$ & $\begin{array}{l}\text { Jeune } \\
\text { Age }\end{array}$ & $\mathbf{R}$ & D & $15 \mathrm{~mm}$ & AL & Epistaxiog \\
\hline $\begin{array}{c}6 \\
1998\end{array}$ & $\begin{array}{l}36 \\
F\end{array}$ & $\begin{array}{l}\text { Jeune } \\
\text { dye }\end{array}$ & $\begin{array}{l}\text { Trobbies du } \\
\text { taractibe }\end{array}$ & $\mathrm{D}$ & $30 \mathrm{~mm}$ & $A G$ & - \\
\hline $\begin{array}{c}7 \\
1996\end{array}$ & 28 & $?$ & $\mathrm{R}$ & G & $20 \mathrm{~mm}$ & AL & . \\
\hline $\begin{array}{c}8 \\
1996\end{array}$ & $\begin{array}{l}36 \\
F\end{array}$ & $6 \mathrm{MOIS}$ & $\mathbf{R}$ & D & $30 \mathrm{~mm}$ & AL &. \\
\hline $\begin{array}{c}0 \\
1997\end{array}$ & $\begin{array}{l}38 \\
F\end{array}$ & BANS & $\mathbf{R}$ & $\begin{array}{l}\text { Bila. } \\
\text { óral }\end{array}$ & $30 \times 25 \mathrm{~mm}$ & AL & $\begin{array}{l}\text { Lyso } \\
\text { coloison } \\
\text { osseuse }\end{array}$ \\
\hline $\begin{array}{c}10 \\
1997\end{array}$ & $\begin{array}{c}45 \\
F\end{array}$ & $\begin{array}{c}\text { Jeune } \\
\text { toe }\end{array}$ & $\mathbf{F}$ & D & $15 \mathrm{~mm}$ & AL & . \\
\hline 11 & $\begin{array}{c}24 \\
F\end{array}$ & 3 & R & D & $20 \mathrm{~mm}$ & AL & - \\
\hline $\begin{array}{c}12 \\
2000\end{array}$ & ${ }_{M}^{12}$ & 2 ANS & $\mathrm{R}$ & D & $15 \mathrm{~mm}$ & AL & - \\
\hline $\begin{array}{c}13 \\
2000\end{array}$ & $\begin{array}{l}13 \\
M\end{array}$ & 5 ANS & A & G & $20 \mathrm{~mm}$ & AG & epistaxis: \\
\hline 2000 & $\begin{array}{l}25 \\
F\end{array}$ & ? & $\mathbf{F}$ & D & $\begin{array}{c}25 \mathrm{~mm} \\
\text { vegetal (pepin) }\end{array}$ & $\mathrm{AL}$ &. \\
\hline $\begin{array}{c}15 \\
2001\end{array}$ & $\begin{array}{l}61 \\
\mathrm{M}\end{array}$ & $?$ & $\begin{array}{l}\text { ON } \\
\mathrm{R}\end{array}$ & c & $50 \times 40 \times 30 \mathrm{~mm}$ & $\mathrm{AOG}$ & - \\
\hline $\begin{array}{c}16 \\
2003\end{array}$ & $\begin{array}{c}14 \\
F\end{array}$ & 2 ANS & $\begin{array}{c}\mathrm{R} \\
\text { EPISTAXIS }\end{array}$ & D & $16 \mathrm{~mm}$ & $A B$ & . \\
\hline $\begin{array}{c}17 \\
2004\end{array}$ & $\stackrel{7}{F}$ & 2ANS & $\begin{array}{c}\text { ON } \\
R \\
E\end{array}$ & D & $15 \mathrm{~mm}$ & AB & . \\
\hline $\begin{array}{c}18 \\
2004\end{array}$ & $\stackrel{11}{F}$ & 3ANS & ON & D & $20 \mathrm{~mm}$ & AG & - \\
\hline $\begin{array}{c}19 \\
2005\end{array}$ & $\begin{array}{l}18 \\
F\end{array}$ & 4 ANS & $\underset{R}{O N}$ & D & $12 \mathrm{~mm}$ & AG & - \\
\hline $\begin{array}{l}20 \\
2006\end{array}$ & $\begin{array}{l}50 \\
M\end{array}$ & $?$ & ON & G & $35 \mathrm{~mm}$ & $A G$ & - \\
\hline
\end{tabular}

$\mathrm{F}$ : féminin.

$R$ : rhinorrhée fétide

$M$ : masculin.

E : épistaxis

AG : anesthésie générale.

Tableau $N^{\circ} \mathrm{I}:$ Résumé des observations cliniques.

La radiographie standard montrait le rhinolithe sous forme d'une image arrondie ou ovoïde de densité calcique avec dans un cas un corps étranger métallique (vis).

(Figure $\mathrm{n}^{\circ} 1-2$ ).

Le scanner (réalisé dans 7 cas) avait donné plus de précision sur le siège exact, les dimensions, la structure du rhinolithe en montrant chez une patiente un rhinolithe bilatéral par perméation trans septale (Figure $n^{\circ} 3$ ).

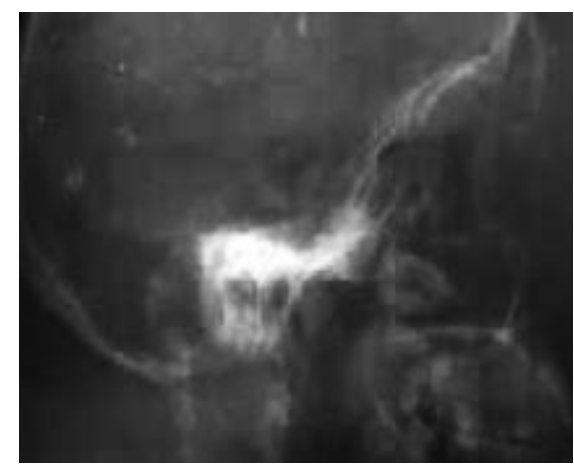

Fig. 1: Radiographie : crâne de profil .

rhinolithe à la partie postérieure de la fosse nasale (Observation $n^{\circ} 9$ ). 


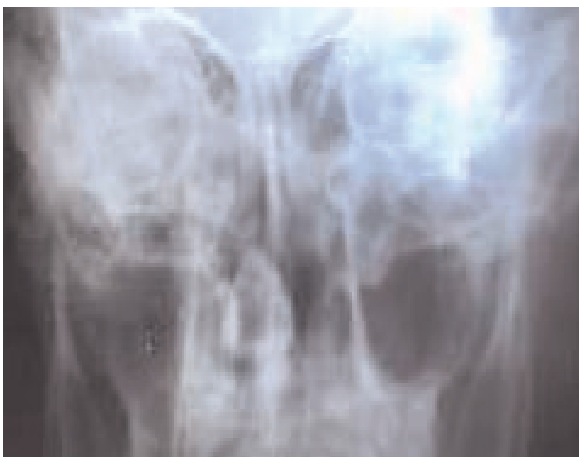

Fig. 2: Radiographie : crâne de face rhinolithe de la fosse nasale droite (Observation $n^{\circ} 9$ )

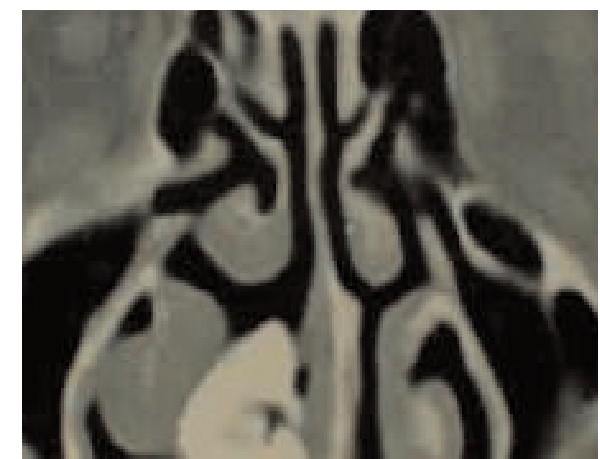

Fig. 3: Scanner : incidence Coronale rhinolithe de la fosse nasale droite : espace inter septo turbinal (Observation $n^{\circ} 18$ )

L'extraction du rhinolithe par voie endonasale était le traitement habituel en utilisant selon les cas ; un crochet, une pince ou l'aspiration nasale.

Les modalités de l'anesthésie étaient une application de xylocaine naphtazolinée à $5 \%$ sous forme d'anesthésie locale de contact potentialisée par une pulvérisation endonasale au vilbiss dans 12 cas $(60 \%)$.

Dans 8 cas $(40 \%)$ on a eu recours à une anesthésie générale avec intubation oro-trachéale. C'était notamment le cas des formes géantes (plus de $30 \mathrm{~mm}$ ) ou de patients pusillanimes. (Tableau I)

Le rhinolithe après extraction a fait l'objet d'un examen précis avec mensuration et recherche d'un corps étranger central.

Pour les 20 rhinolithes le diamètre moyen était de 22,5 $\mathrm{mm}$ avec des extrêmes entre 5 et $50 \mathrm{~mm}$. Une patiente avait deux rhinolithes associés de $5 \mathrm{~mm}$. Cinq formes géantes ont été notées (entre $30-50 \mathrm{~mm}$ de diamètre) (Figure $n^{\circ} 4-5-6$ ).

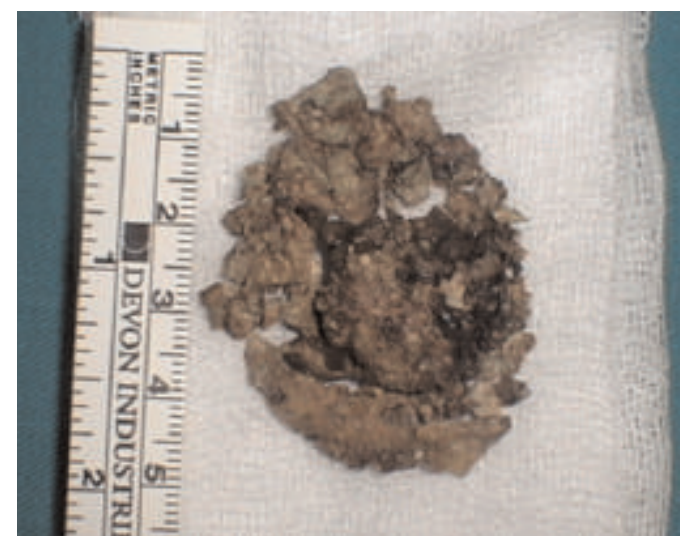

Fig. 4: Rhinolithiase : aspect après extraction : $50 \mathrm{~mm}$ (grand axe) (Observation $\left.n^{\circ} 15\right)$

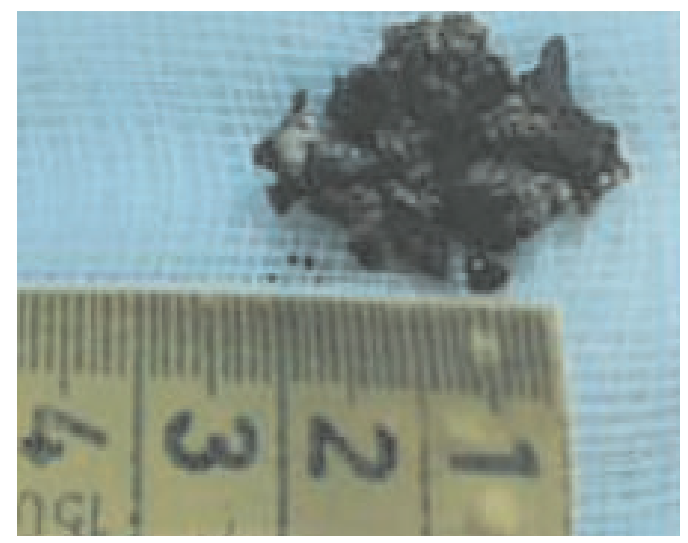

Fig. 5 : Rhinolithiase : aspect après extraction : $25 \mathrm{~mm}$ (grand axe). (Observation $n^{\circ} 7$ )

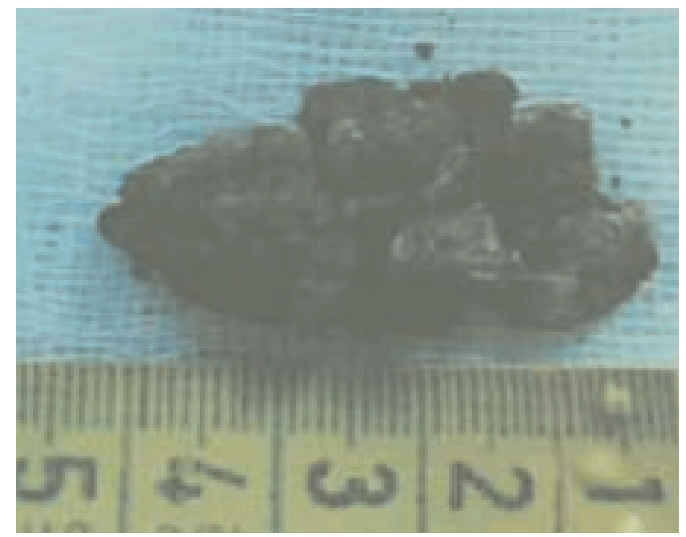

Fig. 6 : Rhinolithiase : aspect après extraction : $40 \mathrm{~mm}$ (grand axe). (Observation $n^{\circ} 9$ )

Sur les 20 cas seuls 3 corps étrangers ont été retrouvés $(15 \%)$ : perle synthétique, vis métallique, pépin de fruits. Les suites étaient favorables dans la majorité des cas. Une épistaxis d'origine septale a été notée dans 2 cas ayant nécessité un simple méchage. 
Aucune récidive ne fut notée après un recul moyen de 16 mois (6 mois -11 ans).

\section{DISCUSSION}

La rhinolithiase est peu fréquente, en particulier dans les pays développés ou elle est souvent découverte fortuitement à l'occasion d'un examen systématique (endoscopie nasale ou d'une imagerie (pour un problème dentaire).

On estime à 3 nouveaux cas/an l'incidence des rhinolithiases dans les pays sous-développés en absence de statistiques fiables.

Sur le plan historique la première description remonte à 1654 par BARTHOLIN (7). En 1943 POLSON avait colligé 380 cas dans la littérature (8).

Parmi les séries les plus importantes; celle d'ELIACHAR en 1970 avec 8 cas (7), CELIANNECK en 199712 cas (5); KHAROUBI en 2004 avec 18 cas (9).

Actuellement on peut estimer entre 650 et 700 le nombre de cas publiés.

Les principales publications sont résumées dans le Tableau II.

\begin{tabular}{|c|c|c|}
\hline AUTEURS & ANNEE & OBSERVATIONS \\
\hline BARTHOLIN (7) & 1654 & 1ère DESCRIPTION \\
\hline POLSON CJ (ع) & 1943 & COLLIGE 387 CAS \\
\hline ABU-JAUDEH_GN (22) & 1951 & RHINOLITHE GEANT $116 \mathrm{gI}$ \\
\hline COWI.H (42) & 1954 & $2 \mathrm{CAS}$ \\
\hline CARROGIOAA $(20)$ & 1966 & RHINOLITHIASE BILATERALE \\
\hline ELIACHAR.I $\langle 7\rangle$ & 1970 & $8 \mathrm{CAS}$ \\
\hline BLESHMAN.M (17) & 1974 & 2 GAS \\
\hline ANTOINE.CG $\langle 19\rangle$ & 1978 & RHINOLITH IASE BILATERALE \\
\hline BOBROV.VM (48) & 1990 & 4 CAS \\
\hline STONEY.P (15) & 1991 & 3 CAS \\
\hline MANIPOUD.P $\{33\}$ & 1994 & $2 \mathrm{CAS}$ \\
\hline KHAROUBI S (1) & 1995 & 3 CAS \\
\hline MOULONGUET.L (38) & 1995 & 2 CAS \\
\hline CELIKKANAT_S (5) & 1997 & $12 \mathrm{CAS}$ \\
\hline KHAROUBI.S (16) & 1998 & $2 \mathrm{CAS}$ \\
\hline MAHTAR.M (2) & 2001 & 5 CAS \\
\hline HADI.V $\{47\rangle^{\prime}$ & 2002 & $8 \mathrm{CAS}$ \\
\hline KHAROUBIS S $^{\prime}(9)$ & 2004 & $18 \mathrm{CAS}$ \\
\hline
\end{tabular}

Tableau N ${ }^{\circ}$ II : Rhinolithiases : principales publications de la littérature

Notre étude actuelle (20 cas) représente la plus importante série publiée dans la littérature.

La pathogénie de la rhinolithiase demeure méconnue. On décrit classiquement deux hypothèses. Le mode exogène ou la précipitation des sels calcaires se fera autour d' un corps étranger et le modèle endogène où le dépôt de ces sels se fait autour de substances et agrégat de la muqueuse pituitaire (sécrétions épaisses, caséification, produits de lyse et desquamation cellulaire) (1).

La symptomatologie clinique de la rhinolithiase n'est pas spécifique et assez souvent négligée. Une rhinorrhée purulente est habituellement retrouvée.

Elle est souvent associée à une obstruction nasale. L'évolution prolongée (parfois des années) des symptômes et leur caractère unilatéral sont de bons éléments d'orientation.
Une épistaxis et des algies faciales par névralgies ou surinfections sinusiennes sont possibles. Les formes asymptomatiques de découverte fortuite : examen ORL, soins bucco-dentaires, bilans radiologiques du massif facial sont fréquemment cités dans la littérature $(10,11$, 12).

Plus rarement on note des formes ophtalmologiques : larmoiement, conjonctivites à répétition voire des troubles neuropsychiques, irritabilité, isolement, tendance dépressive avec difficultés socio-professionnelles (9).

Enfin certaines rhinolithiases ont été décrites dans les suites d'une chirurgie : dacryorhinostomie (13), septoplastie (sur filaments textiles) voire dans les suites d'accidents de la voie publique (fragments de verre, structures et accessoires de voitures) (14).

L'examen endonasal après aspiration des sécrétions nasales et applications de vaso-constricteurs ( xylocaine naphtazolinée à 5\%) est la clé du diagnostic (15).

La rhinoscopie antérieure permet de reconnaître facilement les rhinolithes de la partie antérieure des cavités nasales. Les formes postérieures ou associées à une anomalie turbino-septale (déviation de cloison nasale, hypertrophie turbinale en particulier de la tête du cornet inférieur, une volumineuse concha bullosa) peuvent passer inaperçues. L'endoscopie endonasale (optique rigide $4 \mathrm{~mm}$ ) offre au clinicien des possibilités diagnostiques optimales (9).

Le rhinolithe se présente sous forme d'une masse noire ou jaune - grisâtre à surface spiculée caractéristique occupant le plancher de la cavité nasale (méat inférieur ou l'espace inter-turbino-septal). Le toucher au stylet montre le caractère très dur (pierreux) avec parfois des crépitations de type métalliques (16).

L'endoscopie permet en outre un bilan complet des lésions associées : hypertrophie des cornets, inversion de courbure, déviation postérieure, un granulome ou polype endonasal).

L'imagerie est un apport très apprécié dans le diagnostic et la prise en charge des rhinolithiases (17). Le scanner des cavités naso sinusiennes notamment en coupes coronales montre le rhinolithe sous forme d'une opacité de densité calcique (en totalité ou avec un centre clair). II précise sa forme, son siège exact et ses mensurations. II aide parfois à reconnaître son origine (corps étranger métallique, dent ectopique nasale) (13).

Le scanner est également très utile au choix du procédé thérapeutique le mieux adapté (voie d'abord, prévision sur les difficultés d'extraction).

Les rhinolithiases peuvent présenter des particularités en rapport avec :

- Le nombre : rhinolithiases multiples (plusieurs rhinolithes dans la même cavité nasale -cas $\left.N^{\circ} 3\right)$ (9).

-le siège : rhinolithiase bilatérale vraie ou après lyse du septum ostéo-cartilagineux. $(18,19,20)$.

-le caractère récidivant : les rhinolithiases récidivantes 
sont exceptionnelles. Elles peuvent se développer sur des corps étrangers à répétition (enfant ou arriérés mentaux).

- les dimensions : la forme est définie à partir de $30 \mathrm{~mm}$ de diamètre.

-le poids du rhinolithe : il est très est variable entre 2 et 5 gr $(14,21)$. Un rhinolithe de $116 \mathrm{~g}$ a été décrit par ABU JAUDEH (22).

-les formes associées : une rhinolithiase nasale stricte peut s'associer à une forme sinusienne (antrolithiase) plutôt dans les formes supposées comme d'origine endogènes $(9,23,24,25,26,27,28)$, à une mycose (aspergillose sinusienne)(29) à une tumeur bénigne ou maligne voire à une malformation nasale (Imperforation choanale) $(30,31)$.

L'évolution spontanée est marquée par la persistance des symptômes (rhinorhée fétide et obstruction nasale) avec surinfections naso sinusiennes, épistaxis. Une déformation nasale a été décrite (développement du rhilolithe) de même que des troubles olfactifs (hyposmie, cacosmie) (32, 33, 34, 35).

Une élimination spontanée (petit diamètre ou rhinolithe débutant) est possible à l'occasion d'un éternuement ou d'un mouchage.

Des complications peuvent survenir essentiellement infectieuses ou trophiques : rhinite vestibulaire, furoncle nasal, sinusite (maxillaire, ethmoïde), perforation septale, communication bucco-sinusienne $(18,36,37,38)$.

Elles sont parfois graves ; abcès palpébral, phlegmon de l'orbite, méningite $(2,9,39)$.

Le diagnostic différentiel comprend surtout les tumeurs naso sinusiennes à densité calcique : ostéome, fibrome ossifiant, ostéosarcome, chondrosarcome ; un polype calcifié ou un séquestre osseux d'ostéomyélite (syphilis, radionécrose).

L'extraction du rhinolithe par les voies naturelles résume l'attitude thérapeutique. $(40,41,42)$. Une anesthésie locale de contact renforcée par une pulvérisation de xylocaine naphtazolinée à $5 \%$ est généralement suffisante.

Des particularités en rapport avec le rhinolithe (forme géante ou enclavée dans la partie postérieure des fosses nasales, déviation septale gênante) ; le patient (enfant, sujet pusillanime) ou les lésions associées (sinusite, polype, rhinite hypertrophique majeure, mycose) peuvent nécessiter le recours à une anesthésie générale de préférence avec intubation (chute du rhinolithe ou ses fragments dans les voies aériennes).

On utilise en général un crochet adapté en ramenant le rhinolithe progressivement d'arrière en avant. Une pince à corps étranger ou une aspiration peuvent être utilisés. Le recours à l'endoscopie aux optiques rigides permet une extraction dans de bonnes conditions. Des manoeuvres laborieuses ou répétées (volume du rhinolithe) peuvent nécessiter la mise en place d'une lame de silastic solidarisée au septum nasal pendant 15 jours pour prévenir les éventuelles synéchies nasales.

Un méchage de deux jours est habituel associé à une désinfection nasale de 8 jours avec ou sans antibiothérapie post opératoire.

Un abord chirurgical par voie externe est exceptionnel et dépend surtout des conditions de présentation du rhinolithe dans la cavité nasale : rhinolithe géant incarcéré, malformation turbino-septale gênant l'extraction par les voies naturelles, réaction granulomateuse massive englobant le rhinolithe, anatomie particulière vestibulo-nasale. Cet abord externe peut se faire par voie de rhinotomie sous labiale, allotomie ou d'un Degloving $(9,38)$.

Après extraction on procède à l'expertise physico-chimique du rhinolithe (Mensuration, pesée, recherche d'un corps étranger central et étude Histo-biochimique) (3).

La lithotripsie bien que décrite par certains auteurs ne constitue pas un standard thérapeutique dans ce genre d'affection. Elle suppose une instrumentation adaptée à l'anatomie endonasale de même que le coût et le protocole d'application (plusieurs séances) rend ce procédé peu pratique voire aléatoire réservé au domaine de la recherche clinique (43).

\section{CONCLUSION}

La rhinolithiase est une affection rare de l'adulte de sexe féminin évoluant souvent dans des conditions socio-économiques défavorables pauci symptomatique réalisant le tableau d'une rhinite obstructive sanguinolente et purulente fétide évoluant sur de nombreuses années.

Elle est essentiellement primitive. Dans certains cas le rhinolithe se développe autour d'un corps étranger nasal négligé ou méconnu $(44,45)$.

L'approche diagnostique est simple et repose sur l'examen endonasal en particulier à l'optique rigide. La tomodensitométrie des cavités naso sinusiennes (coupes coronales) affine le diagnostic et offre une iconographie d'un grand apport pré thérapeutique.

Certains aspects inhabituels : forme tumorale bénigne ou maligne, complication révélatrice (phlegmon de l'orbite, ethmoidite aiguë), formes associées (malformation naso sinusienne, dent nasale ectopique) sont à l'origine de certaines publications assez originales (46).

L'extraction se fait le plus souvent par les voies naturelles et les suites sont généralement favorables. Les récidives sont inhabituelles $(47,49,50)$. 


\section{REFERENCES}

1) KHAROUBI. S

Rhinolithiase : à propos de 3 cas Rev Laryngol Otol rhinol (BORDEAUX) 1995; 116 (3) : 223-224.

2) MAHTAR.M - RAJI.A - TARY.M et al.

Rhinolithiase- à propos de 5 cas. J Fr d'ORL 2001; 50 (4):187-190.

3) VINK. BW- VAN HASSELT .P, WORMALD R

A case of rhinolithiasis in botswana: a mineralogical, microscopic and chemical study. J Laryngol Otol. $2002 ; 116(12): 1036-40$.

4) KOUASSI.B - BAMBA. M - ETTE.A ET AL

La rhinolithiase - une entité pathologique nasale oubliée : à propos d'un cas

Les cahiers d'ORL 1988; Tome XXIII (7) 501-503.

5) CELIKKANAT.S - TURGUT.S - OZCAN.P - BALYAN.FR - OZDEM.C

Rhinolithiasis. Rhinology 1997 ; 351 ; 39-40.

6) DAVY - CHEDAUTE.F - JEZEQUEL. JA

Corps étranger des fosses nasales - rhinolithiase EMC ORL 1994 Tome 3 20390 A-10 4P

7 ) ELIACHAR. I - SCHALIT. M

Rhinolithiasis : Report of eight cases Arch oto laryngol;1970;91: $88-90$

8 ) POLSON. C.J

On rhinoliths J. Laryngol otol; 1943; 58: 79-116

9) KHAROUBI.S

Rhinolithiase: étude générale à propos de 18 cas et revue de la littérature.

Cahiers d'ORL 2004 Tome XXXV (4) : 228-234.

10) CARDER.HM - HILL. JJ

Asymptomatic rhinolith : a brief review of the literature and case report Laryngoscope 1966; $76: 524-530$

11) MAHLER.D - KAUFMAN.T

Asymptomatic rhinolith in rhinoplasty : case report Plast reconst surg 1974; 54: 490-491

12 ) COHEN. MA - PACKOTA.GV- STEIN BERG. J

Large asymptomatic antrolith of the maxillary sinus Oral surg - oral med - oral pathol 1991;71:155-7

13 ) V. Kaushik, K. Bhalla, A. Pahade,

Rhinolithiasis Ear, Nose Throat J. 83; $2004: 512,514$

14 ) BERTRAND.B - AOUADI.A - CARRAT.X - TRAISSAC.L

Rhinolithiase géante ; à propos d'un cas Journ FR d'ORL 1995 ; 44 (5) : 347349.

15 ) STONEY.P - BINGHAM.B - OKUDA. I - HAWKE.M

Diagnosis of rhinoliths with endoscopy J. Oto. laryngol 1991, 6, 408-411.

16) KHAROUBI.S

Rhinolithiase : A PROPOS DE DEUX NOUVELLES OBSERVATIONS Journ FR d'ORL $1998: 47$ (2) :115-116.

17) BLESHMAN.M - BONAK-DARPOUR.A - RONIS.

rhinolithiasis: a report of 2 cases Radiology 1974: 113, 615-617.

18 ) KHAROUBI.S

Rhinolithiasis associated with septal perforation. A case report Acta Oto-rhinolaryngologica Belgica: 1998; $52: 241-245$.

19 ) ANTOINE.CC - GERHART.SS - GREGORY. LK

Bilateral rhinolithiasis. Ear Nose ThroatJ. 1978; 57; 50.

20 ) CAROGGIO. A

Bilateral rhinolithiasis Clin oto laryngol; 1966; 18, 253-260.

21 ) PRICE. NI - BATNITZKY . S - KARLIN. CA - NORRIS. CW

Giant nasal rhinolith Am J Neuro radiol: 1981;2, 371-373.

22 ) ABU - JAUDEH.CN

A giant rhinolith Laryngoscope : 1951; 61: 271-7.

23) MANJALY. G - PAHOR. AL

Antral rhinolithiasis and tooth filling Ear nose and throat Journal: 1994; 9; 676679.

24 SALGADO AVILA. C - AZ CARATE RANGEL. C

Rhinolithiase Rev Laryngol Otol Rhinol (BORDEAUX) 1995; 116 (3): 221-222. 25) ISHIYAMA. T

Maxillary antrolith : report of a case Auris nasus larynx; 1988; 15, 185.

26) IRIS.LE - GRAY. RP - SORENSON.FM

Antrolith Oral surg - Oral med - Oral Pathol; 1990; 70 : 682-3.
27) - BOWERMAN. JE

The maxillary antrolith J. Laryngol otol; 1969; $83: 873-882$.

28) - DAVIS.O - WOLF. C.A

Rhinolithiasis and maxillary antrolithiasis EAR nose throat J; 1995; 64: 421

29) - OGATA. Y - OKINAKA. Y - TAKAHASHI.M

Antrolith associated with aspergillosis of the maxillary sinus : report of a case

J. Oral maxillo fac surg; 1997; $55: 1339-1341$.

30) - ALI. DS

Congenital unilateral choanal atresia associated with a rhinolith

J. Laryngol otol 1967; 81, 359-362.

31) WORGAN. D

Unilateral choanal atresia associated with a rhinolith

$J$. Laryngol otol 1966; $80: 418-421$.

32) LOVEWELL.L

Comment on "An unexpected cause of halitosis "BR Dent J; 1984; 157: 384. 33) MANIPOUD . P - GUICHARD.C - LAFAYE. M

Rhinolithase à propos de deux cas et revue de la littérature Journ FR D'ORL 1994; 3 : 133-135.

34) VERGES - LLARDENT. $P$

Rhinolithasis Acta oto rinolaryngol iber am; 1965; $16: 522-531$

35) RIPP.G

Rhinolith Oral surg - oral med - oral pathol; 1968: 26-43.

36) FLOODT .TR

Rhinolith : an unusual cause of palatal perforation BR. J Oral maxillo facial surg 1988; 26 : 486- 90.

37) GILL.RS - LAL. M

Perforation of the hard plate by a Rhinolith and its repair J. Laryngol otol 1977; 91 (35) : 85-89.

38) MOULONGUET.L - BRETTE. MD - MONTEIL. JP

Deux cas d'obstruction nasale unilatérale liée à une rhinolithiase Ann oto-laryngol (PARIS) 1995; 112(8): 406-409.

39) SACKO. HB

Rhinolithiase : a propos d'une observation et revue de la littérature Lettre d'ORL; 1995; 201; 17-18.

40) HUNT.MJ - COTTON.BP - JOSEPH. DJ

Rhinoliths Arch otorhinolaryng; 1966; $83: 550-560$

41) EZSIAS.A - SUGAR. AW

Rhinolith : An unusual case and update Ann otol rhinol laryngol; 1997; 106: 135-138.

42) COWIE. H

Two sysmptomless rhinoliths J. Laryngol otol; 1954; 68: 250-255.

43) A.Mink, I. Gati, J. Szekely

Nasolith removal with ultrasound lithotripsy, HNO (1991); 39 : 116 -117.

44) MATKARIMOV.R

Arhinolith with nucleus from the remains of a gauze tampon Vest - otorhinolaryngol; Juill - Aout 1992; $4: 32$.

45) J. Levy, T. Monos, M. Puterman

Rhinolithiasis: a very late complication after dacryocystorhinostomy with rubbergum and polyethylene stenting, Am. J. Opthalmol. 138; (6); (2004): 1065 -1067. 46) MEYER . JR - QUINT. DJ

Post traumatic rhinolith AM J Neuro radiol 199314 ; (5) : 1181-1182.

47) MARINESCU. C

recurrent rhinolithiasis.

Rev Chir Oncol Radiol ORL $1978 ; 23$ (2):149-51.

48) BOBROV. VM

4 cases of nasal calculi Vestnik oto rhino laringol; $1990: 69-71$

49) HADI. U- GHOSSAINI. S- ZAYTOUN. G.

Rhinolithiasis: a forgotten entity. Otolaryngol Head Neck Surg. 2002;126(1):48-

50) BALATSOURAS. D - EILIOPOULOS. - KABEROS. A - ECONOMOU. C. Rhinolithiasis: an unusual cause of nasal obstruction. Rhinology. 2002;40(3):162-4 